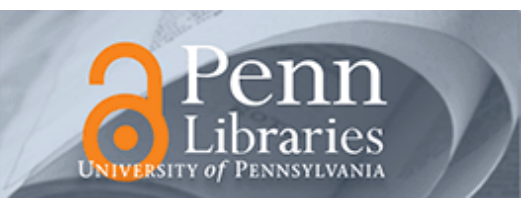

University of Pennsylvania ScholarlyCommons

\title{
Temperature dependence of mechanical stiffness and dissipation in ultrananocrystalline diamond
}

\author{
Vivekananda P. Adiga \\ University of Pennsylvania, adiga@seas.upenn.edu
}

A V. Sumant

Argonne National Lab

S Suresh

Innovative Micro Technology

C Gudeman

Innovative Micro Technology

RoAdwietl| and additional works at: https://repository.upenn.edu/mse_papers

Iryonne National Lab

Part of the Condensed Matter Physics Commons, and the Materials Science and Engineering

\section{Commons}

See next page for additional authors

\section{Recommended Citation}

Adiga, V. P., Sumant, A. V., Suresh, S., Gudeman, C., Auciello, O., Carlisle, J. A., \& Carpick, R. W. (2009). Temperature dependence of mechanical stiffness and dissipation in ultrananocrystalline diamond.

Retrieved from https://repository.upenn.edu/mse_papers/173

Copyright 2009 Society of Photo-Optical Instrumentation Engineers. Reprinted from:

Temperature dependence of mechanical stiffness and dissipation in ultrananocrystalline diamond V. P. Adiga, A. V. Sumant, S. Suresh, C. Gudeman, O. Auciello, J. A. Carlisle, and R. W. Carpick, Proc. SPIE 7318, 731818 (2009), DOI:10.1117/12.822795

URL: http://dx.doi.org/10.1117/12.822795

One print or electronic copy may be made for personal use only. Systematic reproduction and distribution, duplication of any material in this paper for a fee or for commercial purposes, or modification of the content of the paper are prohibited.

This paper is posted at ScholarlyCommons. https://repository.upenn.edu/mse_papers/173

For more information, please contact repository@pobox.upenn.edu. 


\title{
Temperature dependence of mechanical stiffness and dissipation in ultrananocrystalline diamond
}

\begin{abstract}
Ultrananocrystalline diamond (UNCD) films are promising for radio frequency micro electro mechanical systems (RF-MEMS) resonators due to the extraordinary physical properties of diamond, such as high Young's modulus, quality factor, and stable surface chemistry. UNCD films used for this study are grown on $150 \mathrm{~mm}$ silicon wafers using hot filament chemical vapor deposition (HFCVD) at $680^{\circ} \mathrm{C}$. UNCD fixed free (cantilever) resonator structures designed for the resonant frequencies in the $\mathrm{kHz}$ range have been fabricated using conventional microfabrication techniques and are wet released. Resonant excitation and ring down measurements in the temperature range of $138 \mathrm{~K}$ to $300 \mathrm{~K}$ were conducted under ultra high vacuum (UHV) conditions in a custom built UHV AFM stage to determine the temperature dependence of Young's Modulus and dissipation (quality factor) in these UNCD cantilever structures. We measured a temperature coefficient of frequency (TCF) of 121 and $133 \mathrm{ppm} / \mathrm{K}$ for the cantilevers of $350 \mathrm{im}$ and 400 ìm length respectively. Young's modulus of the cantilevers increased by about $3.1 \%$ as the temperature was reduced from $300 \mathrm{~K}$ to $138 \mathrm{~K}$. This is the first such measurement for UNCD and suggests that the nanostructure plays a significant role in modifying the thermo-mechanical response of the material. The quality factor of these resonators showed a moderate increase as the cantilevers were cooled from $300 \mathrm{~K}$ to $138 \mathrm{~K}$. The results suggest that surface and bulk defects significantly contribute to the observed dissipation in UNCD resonators.
\end{abstract}

\section{Keywords}

resonators, dissipation, modulus, ultrananocrystalline diamond

\section{Disciplines}

Condensed Matter Physics | Materials Science and Engineering

\section{Comments}

Copyright 2009 Society of Photo-Optical Instrumentation Engineers. Reprinted from:

Temperature dependence of mechanical stiffness and dissipation in ultrananocrystalline diamond V. P. Adiga, A. V. Sumant, S. Suresh, C. Gudeman, O. Auciello, J. A. Carlisle, and R. W. Carpick, Proc. SPIE 7318, 731818 (2009), DOI:10.1117/12.822795

URL: http://dx.doi.org/10.1117/12.822795

One print or electronic copy may be made for personal use only. Systematic reproduction and distribution, duplication of any material in this paper for a fee or for commercial purposes, or modification of the content of the paper are prohibited.

\section{Author(s)}

Vivekananda P. Adiga, A V. Sumant, S Suresh, C Gudeman, O Auciello, J A. Carlisle, and R W. Carpick 
Invited Paper

\title{
Temperature dependence of mechanical stiffness and dissipation in ultrananocrystalline diamond
}

\author{
Authors : \\ V.P. Adiga ${ }^{1}$, A. V. Sumant ${ }^{2}$, S. Suresh ${ }^{3}$, C. Gudeman ${ }^{3}$, O. Auciello ${ }^{2}$, J. A. Carlisle ${ }^{4}$, R.W. \\ Carpick $^{5}$ \\ ${ }^{1}$ Department of Materials Science and Engineering, University of Pennsylvania, ${ }^{2}$ Argonne \\ National Laboratory, ${ }^{3}$ Innovative Micro Technology, ${ }^{4}$ Advanced Diamond Technologies, \\ Department of Mechanical Engineering, University of Pennsylvania
}

\begin{abstract}
:
Ultrananocrystalline diamond (UNCD) films are promising for radio frequency micro electro mechanical systems (RF-MEMS) resonators due to the extraordinary physical properties of diamond, such as high Young's modulus, quality factor, and stable surface chemistry. UNCD films used for this study are grown on $150 \mathrm{~mm}$ silicon wafers using hot filament chemical vapor deposition (HFCVD) at $680^{\circ} \mathrm{C}$. UNCD fixed free (cantilever) resonator structures designed for the resonant frequencies in the $\mathrm{kHz}$ range have been fabricated using conventional microfabrication techniques and are wet released. Resonant excitation and ring down measurements in the temperature range of $138 \mathrm{~K}$ to $300 \mathrm{~K}$ were conducted under ultra high vacuum (UHV) conditions in a custom built UHV AFM stage to determine the temperature dependence of Young's Modulus and dissipation (quality factor) in these UNCD cantilever structures. We measured a temperature coefficient of frequency (TCF) of 121 and 133 $\mathrm{ppm} / \mathrm{K}$ for the cantilevers of $350 \mu \mathrm{m}$ and $400 \mu \mathrm{m}$ length respectively. Young's modulus of the cantilevers increased by about $3.1 \%$ as the temperature was reduced from $300 \mathrm{~K}$ to $138 \mathrm{~K}$. This is the first such measurement for UNCD and suggests that the nanostructure plays a significant role in modifying the thermo-mechanical response of the material. The quality factor of these resonators showed a moderate increase as the cantilevers were cooled from 300 $\mathrm{K}$ to $138 \mathrm{~K}$. The results suggest that surface and bulk defects significantly contribute to the observed dissipation in UNCD resonators.
\end{abstract}

\section{Keyword list}

Temperature dependence of Young's modulus, quality factor, resonators, ultrananocrystalline diamond, dissipation, mechanical properties, grain boundaries, defects, nanocrystalline materials, Debye temperature

\section{INTRODUCTION:}

Diamond is the hardest known material on earth. It exhibits exceptional physical and mechanical properties such as high stiffness, high acoustic velocity, and a stable surface with extraordinary tribological behavior. Mechanical properties of diamond are stable across a wide temperature range. All these properties make it an attractive candidate for applications for microelectromechanical systems (MEMS) and nanoelectromechanical systems (NEMS), such as radio frequency MEMS resonators with high quality factors, and MEMS switches.[1-5] Uniform, conformal ultrananocrystalline diamond (UNCD) thin films (grain size 2-5 nm) are now grown using either the microwave plasma chemical vapor deposition (MPCVD) process or hot filament chemical vapor

Micro- and Nanotechnology Sensors, Systems, and Applications, edited by Thomas George, M. Saif Islam, Achyut K. Dutta, Proc. of SPIE Vol. 7318, 731818 · (c) 2009 SPIE · CCC code: 0277-786X/09/\$18 · doi: 10.1117/12.822795 
deposition (HFCVD) process. These films, despite the presence of a large fraction of grain boundaries containing non-diamond carbon and hydrogen,[6] exhibit mechanical properties close to that of single crystal diamond. The ability to grow uniform UNCD films over a large area (>150 mm wafers) at lower temperatures has now enabled the co-integration of complementary metal-oxide-semiconductor (CMOS) electronics with UNCD devices. [7-9] The temperature dependence of the elastic properties and the dissipation in low temperature-grown UNCD films play an important role in the suitability of these films in RF-MEMS resonator applications. As well, UNCD cantilever probes for AFM applications have recently been developed. Most of these applications necessitate the need to study the temperature dependence of stiffness and dissipation in these films to determine the temperature sensitivity of these devices, such as the force sensitivity of cantilever AFM probes .[10]

We have recently reported mechanical properties and dissipation in HFCVD-grown UNCD cantilevers (growth temperature $680^{\circ} \mathrm{C}$ ). [11] We have measured a Young's modulus of $790 \pm 30 \mathrm{GPa}$ by resonant excitation of the fundamental mode of the cantilevers. Ring down measurements yielded a quality factor in the range of 5000 to 16000. The lower Young's modulus in UNCD micro-cantilevers compared to single crystal diamond is mainly due to the presence of the higher fraction of grain boundaries containing $\mathrm{sp}^{2}$ and $\mathrm{sp}^{1}$ bonded carbon. A discussion of room temperature modulus and dissipation in HFCVD grown UNCD films is provided elsewhere.[11] The dissipation observed is much higher than single crystal silicon cantilevers and polycrystalline diamond cantilevers. Stress assisted relaxation of the defects in the grain boundaries and at the surface are believed to be responsible observed dissipation.[11, 12] The temperature dependence of the resonant frequency (and hence the Young's modulus) and dissipation in UNCD cantilever beams is useful for determining the nature of such defects. In this article we report the temperature dependence of frequency, hence Young's modulus and quality factor in UNCD cantilever beams grown using the HFCVD process.

\section{BACKGROUND}

\subsection{Temperature dependence of resonant frequency:}

The temperature dependence of Young's modulus for crystalline materials can be attributed to the anharmonic effects of lattice vibrations and also to changes in the bond length with temperature. [10,13-15] An analytical solution to the thermally driven softening of Young's modulus has been a challenge. Wachtman et al.[13] developed an empirical solution for temperature dependent modulus as follows:

$$
E(T)=E_{0}-A T \exp \left(-\frac{T_{0}}{T}\right)
$$

where $\mathrm{E}_{0}$ is Young's modulus at $0 \mathrm{~K}$. Watchtman assumed that $\mathrm{A}$ is a temperature independent constant related to the Grueneisen parameter $\gamma$, and $T_{0}$ is characteristic temperature related to Debye temperature, $\Theta_{D}$. Later Anderson et al.[14] derived an equation for the temperature dependence of the bulk modulus by taking into account effects of lattice vibrations.

$$
B_{S}(T)=B_{0}-\frac{\gamma \delta}{V_{0}} 3 R T \times H\left(\frac{\Theta_{D}}{T}\right)
$$

$\mathrm{R}$ is the ideal gas constant, $\mathrm{B}_{0}, \mathrm{~V}_{0}$ is the bulk modulus, volume at $0{ }^{\circ} \mathrm{K}, \delta$ is the Anderson-Grueneisen parameter and $\mathrm{H}(\mathrm{x})$ is given by

$$
H(x)=\frac{3}{x^{3}} \int_{0}^{x} \frac{x^{3}}{e^{x}-1} d x
$$


We can rewrite this equation in terms of specific heat $\mathrm{c}_{\mathrm{v}}$

$$
B_{S}(T)=B_{0}-\frac{\gamma \delta}{V_{0}} \int_{0}^{T} c_{v} d T
$$

All the above relations assume that the change in volume is much less than the change in the modulus with temperature. At very low temperatures $\mathrm{T}<<\Theta_{\mathrm{D}}$, the rate of change of modulus decreases and is zero at $0 \mathrm{~K}$ so as to satisfy Nernst's Law:[10] As indicated in equation (4), the derivative of the temperature dependence of the bulk modulus indicates the temperature dependence of the specific heat of the material. The temperature dependence of the elastic constants for single crystal diamond has been studied[16-18] and theoretically predicted[15] and showed that the rate of change of Young's modulus of single crystal diamond with respect to temperature is dramatically reduced below $160 \mathrm{~K}$.

In nanocrystalline /nano-scale materials the temperature dependence of the modulus can be much different than for single crystals because of the presence of a large proportion of grain boundaries. Recent progress has shown that it is possible to correlate changes in Young's modulus of nano-scale materials to atomistic parameters $[15,19,20]$ by the bond-order-length-strength (BOLS) correlation, by taking into account the bond nature, bond order, bond length and bond strength. Using this method, Gu et al.[20] proposed expressions for the size and temperature dependence of Young's modulus and the Debye temperature. The broken bonds at abrupt interfaces cause the remaining bonds to be stronger and shorter in diamond. According to Gu et al.,[20] the Young's modulus and Debye temperature of nanoscale materials can increase or decrease compared to the bulk depending on the nature of the bonds and temperature. In nanocrystalline materials however, we should take into account the presence of grain boundaries and inter crystalline bonds. In UNCD particular, carbon atoms at the grain boundaries are either threefold / twofold coordinated or form bonds at different lengths or angles from those observed in diamond.[21] As the grain sizes become smaller, there will be more surface atoms compared to the bulk. The presence of $\mathrm{sp}^{1}$ and $\mathrm{sp}^{2}$ bonded carbon at the grain boundaries influences the observed mechanical stiffness and its temperature dependence in UNCD. Even though bonds between two-fold coordinated $\mathrm{sp}^{1}$ atoms or between three-fold coordinated $\mathrm{sp}^{2}$ atoms in their ideal geometric configurations are usually stronger than bonds between fourfold coordinated $\mathrm{sp}^{3}$ atoms, bonds between $\mathrm{sp}^{1}$ or $\mathrm{sp}^{2}$ atoms are stabilized by p-bonding, which is very sensitive to the geometry. As a consequence, the local bulk moduli of $\mathrm{sp}^{1}$ and $\mathrm{sp}^{2}$ atoms could to be significantly lower than the bulk moduli of $\mathrm{sp}^{3}$ atoms.[21]The presence of hydrogen in grain boundaries also influences the nature of bonding. Such weaker bonds bend or stretch with greater ease compared to the bonds in the crystalline grains. Similarly, the presence of grain boundaries lowers the Debye temperature of nanocrystalline diamond due to eliminating the higher frequency acoustic modes. However, in the high temperature limit, the Debye temperature can be estimated by comparing (1) and (4). This yields

$$
A=\frac{R \gamma \delta}{V_{0}}, T_{0} \approx \frac{\Theta_{D}}{2},
$$

assuming that $E_{S} \approx 3 B_{S}$ (Poisson's ratio $v<<1$ ).[10]

\subsection{Dissipation in micro-cantilevers:}

Dissipation in high aspect ratio UNCD cantilevers resonators under UHV conditions is limited by intrinsic dissipation due to the stress-assisted relaxation of the defects at grain boundaries and at surfaces.[11, 12, 22] Impurity atoms, dislocations, and other defects at grain boundaries and at surfaces undergo a transition from one state to another similar to two-level systems when under stress. Dissipation in such systems exhibit Debye peaks with a characteristic relaxation time $\tau$ which is unique to that transition. The frequency dependent quality factor in such systems is given by:[23] 


$$
Q_{\text {Defect }}=A\left[\frac{\omega \tau}{1+(\omega \tau)^{2}}\right]^{-1}
$$

where $A$ is a constant which depends on the nature of the defect and the defect concentration (number of defects per unit volume) and the $\tau$ is the relaxation time which follows the Arrhenius relation given by [23]

$$
\frac{1}{\tau}=\frac{1}{\tau_{0}} \exp \left(-\frac{E_{A}}{k_{B} T}\right)
$$

where $E_{A}$ is the activation energy for the process, $k_{B}$ is Boltzmann's constant, and $1 / \tau_{0}$ is the characteristic atomic vibration frequency. The temperature dependence of dissipation will helpful to observe the presence of dissipation peaks due to defect relaxations.

\section{EXPERIMENTAL SET UP}

UNCD films were grown using the HFCVD technique at $680^{\circ} \mathrm{C}$ on silicon wafers (diameter $=150 \mathrm{~mm}$ ) with predominantly methane/hydrogen growth chemistry. The films are grown by Advanced Diamond Technologies Inc. and are known commercially as Aqua 25. UNCD cantilever resonator structures are fabricated using conventional lithography techniques. The wafer is diced to fit in the AFM sample holder (discussed further below), with each die containing 4 cantilevers as shown in Figure 1. The substrate underneath the cantilever has been etched using $\mathrm{XeF}_{2}$ and cantilevers used for this study have an overhang of $85 \mu \mathrm{m}$. The substrate underneath the cantilever have been cleaved as shown in SEM image shown in Figure 1.
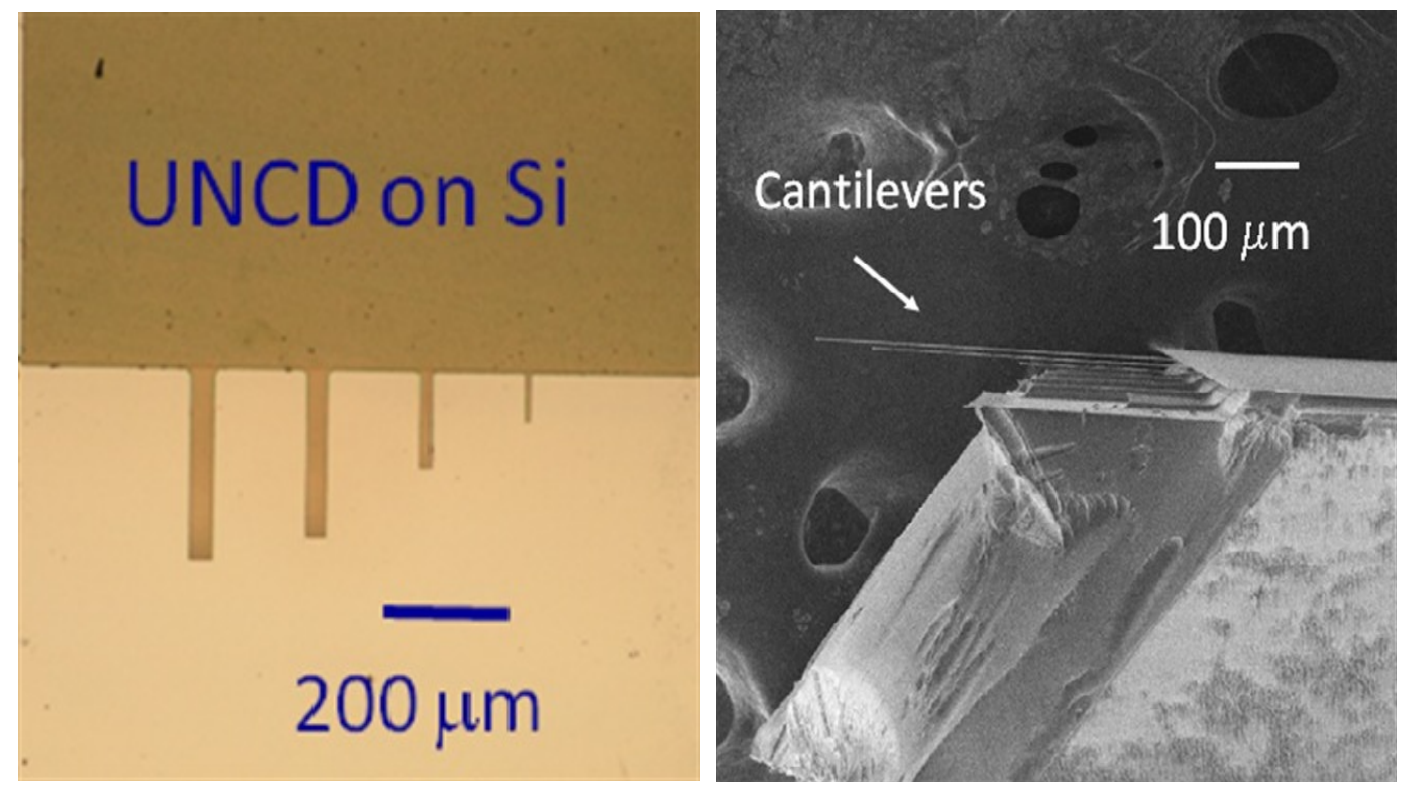

Figure 1. (a) An optical image of the cantilever die. Unreleased cantilever die consisting of four different cantilevers with dimensions ranging from $100 \mu \mathrm{m}$ to $400 \mu \mathrm{m}$ with length-to-width ratio of 7.5, and thickness of $1 \mu \mathrm{m}$. (b) SEM image taken after cleaving the die showing the cantilevers extending from the chip with a small overhang at the cantilever base. 
This avoids the laser signal interference from the more reflective silicon substrate while characterizing the cantilevers in the AFM (RHK UHV 350). Cantilevers are cleaned for any remaining silicon particles and are tested under UHV $\left(2 \times 10^{-10}\right.$ Torr) conditions to avoid condensation of water or any other contaminants during the cooling of the cantilevers. To measure the temperature dependence of modulus and dissipation, a custom built AFM stage has been used with a piezo ceramic glued onto a tilted $\left(22.5^{\circ}\right)$ invar holder. Cantilever dies are mounted on top of the piezo ceramic and the cantilever's deflection is measured using the AFM detection scheme. The fundamental flexural resonance is found by manually scanning the drive frequency. To determine the quality factor, a ring down measurement is done whereby an exponential fit to the ring down curve is performed after halting resonant excitation. This gives a measure of dissipation in the fixed free beams at the flexural resonance. Temperature is measured using a thermocouple attached on top of the UNCD cantilever die. Cooling and warming rates are $1 \mathrm{~K}$ $/ \mathrm{min}$. The characterization of the films, fabrication of UNCD cantilevers and the experimental set up used for the resonant frequency and dissipation measurement are reported elsewhere.[11]

\section{RESULTS AND DISCUSSION:}

\subsection{Temperature dependence of resonant frequency:}

The resonant frequency of the cantilever beams is given by

$$
f_{n}=\frac{\beta_{n}^{2}}{2 \pi} \frac{t}{L^{2}} \sqrt{\frac{E}{12 \rho}}
$$

where $L$ and $t$ are the length and thickness of the cantilever and $E$ and $\rho$ are Young's modulus and density respectively. $\beta_{n}$ is a constant and equal to $\sim 1.875,4.694,7.855$ for $n=1,2,3$ respectively. The temperature dependence of the resonant frequency (fundamental mode) of a typical UNCD cantilever is plotted in Fig. 2. No difference is seen between cooling and heating, and the qualitative behavior is reproducible amongst all of the cantilevers. For all the measured cantilever beams, we observed little change in the resonant frequency below $160 \mathrm{~K}$, followed by a transition to a nearly linear decrease with frequency. Table 1 shows temperature coefficient of frequency (TCF) close to room temperature for 3 different cantilevers.

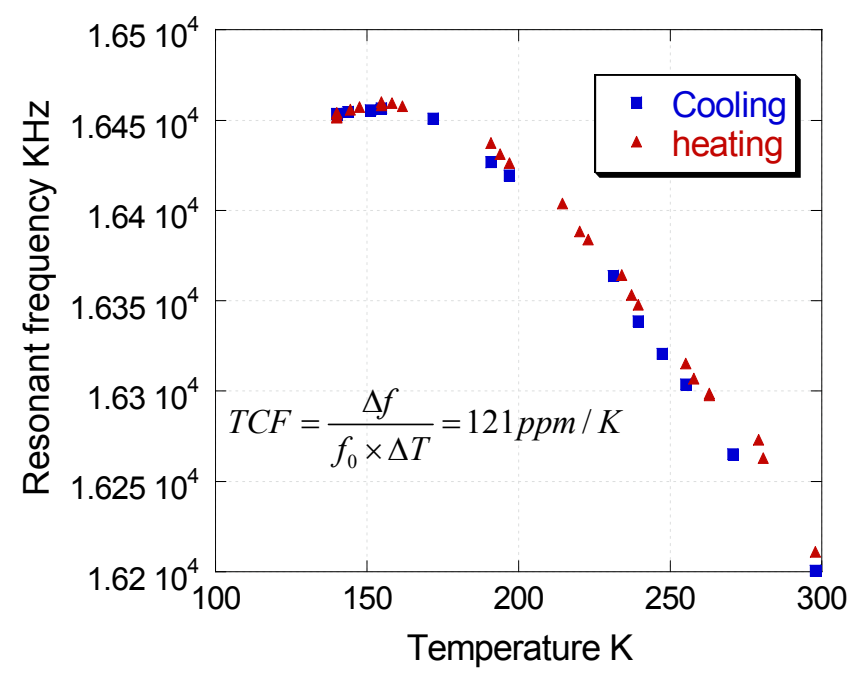

\begin{tabular}{|l|l|}
\hline Cantilever & TCF ppm /K \\
length $\mu \mathrm{m}$ & \\
\hline 400 & -133 \\
\hline 350 & -121 \\
\hline
\end{tabular}

Figure 2. The temperature dependence of frequency of a typical cantilever during cooling and heating at a rate of $\sim 1 \mathrm{~K} / \mathrm{min}$. All the tested cantilevers showed a much lower temperature dependence below $160 \mathrm{~K}$. Table 1 . TCF values for 2 different cantilevers close to room temperature. 
The change in resonant frequency with temperature can be used to calculate the corresponding change in modulus according to Eq. 8. The changes in the dimension of the cantilever due to thermal contraction / expansion and changes in the density with temperature put together contributes to an error of the order of the coefficient of thermal expansion $\left(1 \times 10^{-6} / \mathrm{K}\right.$ for single crystal diamond) and can be neglected. Figure 3 depicts the relative change in the Young's modulus of UNCD micro cantilever beam of $350 \mu \mathrm{m}$ length with an increase in Young's modulus by 3.1 $\%$ in the measured temperature range. References $[12,17,18]$ give the temperature dependence of modulus of single crystal as well as CVD grown diamond of grain sizes ranging from $\sim 400 \mathrm{~nm}[12]$ to $\sim 100 \mu \mathrm{m}$.[18]

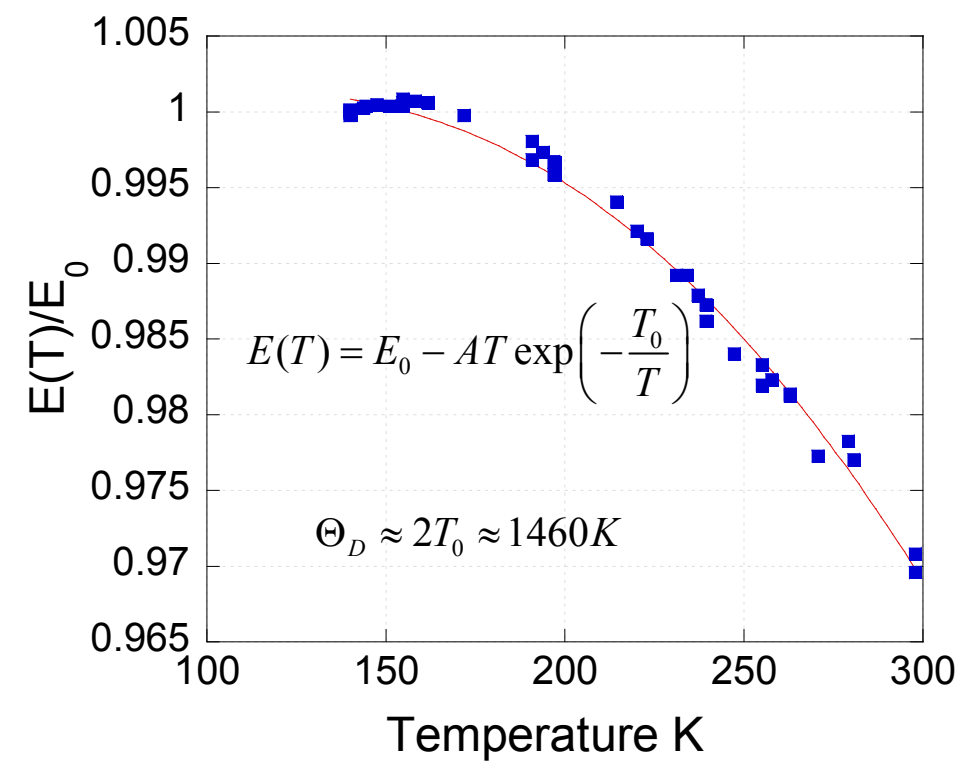

Figure 3. Relative change of Young's modulus with temperature. A curve fit to Wachtman's semi-empirical relation yields a Debye temperature of $1460^{\circ} \mathrm{K}$.

For polycrystalline diamond (grain size $~ 300-500 \mathrm{~nm}$ ) cantilevers Sepúlveda et al.[12] reported a TCF of $25 \mathrm{ppm} / \mathrm{K}$. The temperature dependence of Young's modulus of microcrystalline diamond (MCD)[18] seems to be comparable to our measurements. However, single diamond showed a much lower temperature dependence with a $0.16 \%$ increase in the modulus from $298 \mathrm{~K}$ to $138 \mathrm{~K}$.[16, 17] Reference [15] gives a theoretical prediction of of this increase. We assert that the higher temperature dependence of the modulus in UNCD films is due to the presence of a much higher proportion of grain boundaries containing $\mathrm{sp}^{2}$ and $\mathrm{sp}^{1}$ bonded carbon and the impurities like hydrogen, which form weaker bonds.

Using the curve fit to Wachtman's semi-empirical, we found the Debye temperature for UNCD to be 1460 K. For single crystal diamond Debye temperature is estimated to be 1860 K.[24] Reduction in the Debye temperature of other nanocrystalline materials due to the elimination of high frequency acoustic modes has been observed previously[25] and is consistent with theoretical predictions.[20, 25] However the Debye temperature approximated using Wachtman's semi-empirical relation will be more accurate if we know the temperature dependence of Young's modulus over a wider temperature range, especially the temperature dependence at much higher temperature. For a high temperature material like diamond, $300 \mathrm{~K}$ is still rather low.

It remains to investigate effects of the overhang at the cantilever base on the temperature dependence of resonant frequency of the beams. Residual stresses in the low temperature grown UNCD films cause wrinkles at the underetched cantilever base. Residual stresses are partly due to the thermal stresses which changes as a function of temperature and hence the amplitude and wavelength of the wrinkles.[11] This may affect the stiffness and resonance frequencies measured. Experiments that fully control for this effect are currently underway.

\subsection{Dissipation in UNCD cantilevers}

Ring-down measurements were conducted at the fundamental flexural resonance frequencies of the cantilevers under UHV conditions. The curve fit to the damped oscillations after the resonant excitation ceases yielded quality 
factors in the range of 5000-16000 for all the cantilevers of different dimensions and overhangs, with no specific dependence on any of the dimensions.[11] Considering the dimension of our levers and the absence of any reflective coating on these cantilevers, we conclude that the observed dissipation is attributed to intrinsic dissipation in UNCD beams. Contributions from thermoelastic dissipation (TED) and dissipation due to interaction between thermal phonons and acoustic phonons together accounts for less than $0.1 \%$ of the observed dissipation.[11], indicating that dissipation is mainly due to the relaxation of large number of defects in the film.

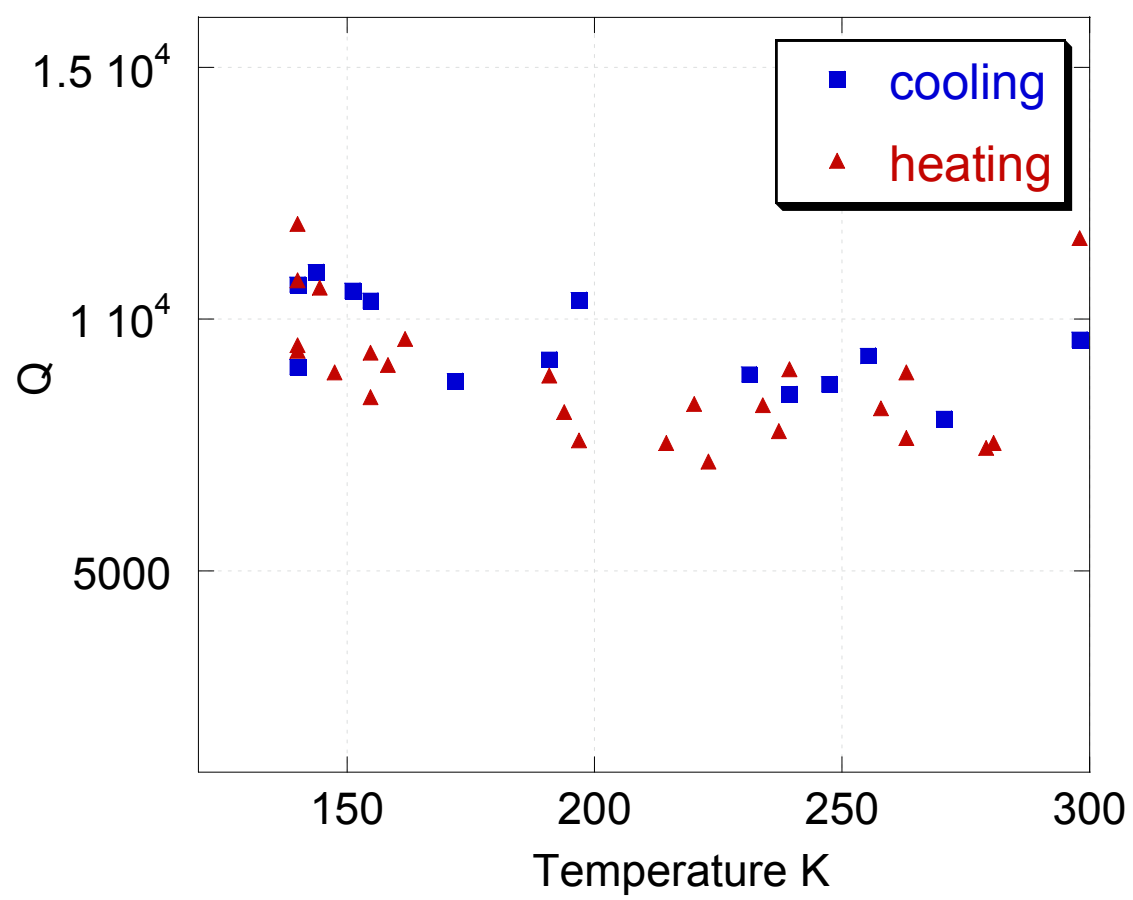

Figure 4: Typical temperature dependence of the quality factor of $350 \mu \mathrm{m}$ long UNCD cantilever beam . A moderate decrease in dissipation (increase in $Q$ ) is observed at lower temperatures.

Figure 4 shows the quality factor as a function of temperature of UNCD for one of the measured cantilever beams of $350 \mu \mathrm{m}$ length. We did not observe the presence of characteristic Debye peaks associated with defects in the measured temperature range. A moderate improvement in quality factor can be seen for all the cantilevers that we measured. Such a measurement over a broader temperature range will determine the factors that dominate the observed dissipation in UNCD beams. Sepúlveda et al. [12,22] has reported a higher quality factor for polycrystalline diamond fixed-free resonators with comparable dimensions, which indicates that higher dissipation in our UNCD resonators is mainly due to the presence of the higher proportion of grain boundaries and defects. Hutchinson et al. [5] measured the temperature dependence of dissipation of metal / nanocrystalline diamond (NCD) composite fixed-fixed beams which showed the presence of a Debye peak at $55 \mathrm{~K}$ and a dramatic increase in dissipation above $100 \mathrm{~K}$. However for these high frequency $(\mathrm{MHz})$ beams they measured a lower quality factor (Q 3000, at room temperature) than the low frequency UNCD cantilever resonators. Higher dissipation in these high frequency resonator beams has been attributed to surface effects and the clamping losses [26, 27] is supported by investigations of the scaling of dissipation with the dimensions of NCD fixed-fixed resonators by Imboden et al[28]. Factors that contribute to the higher dissipation in high frequency resonators may also include TED, and the dissipation in the metal layer of the composite beams.[26]

\section{CONCLUSIONS}

Cantilever resonator structures were fabricated from UNCD grown using a low temperature $\left(680{ }^{\circ} \mathrm{C}\right) \mathrm{HFCVD}$ process. The Young's modulus of the UNCD films increased by $3.1 \%$ as the temperature was cooled $300 \mathrm{~K}$ to 138 $\mathrm{K}$ and rate of increase dropped dramatically below $160 \mathrm{~K}$. Imperfections and impurities (disordered carbon, 
hydrogen, dangling bonds) at the grain boundaries are asserted to cause the reduction in the modulus as well as increase in the temperature dependence of the modulus of UNCD films. The UNCD cantilever resonators exhibited much higher dissipation compared to polycrystalline diamond cantilevers with comparable resonant frequencies. We attribute higher dissipation in our resonators mainly due to the presence of large number of defects and impurities at grain boundaries and surface and dissipation increased moderately with temperature.

\section{ACKNOLEDGEMENTS}

This project was supported by DARPA 06-W238 and DOE, under contract DE-AC02-06CH05117. Use of the Center for Nanoscale Materials was supported by the U. S. Department of Energy, Office of Science, Office of Basic Energy Sciences, under Contract No. DE-AC02-06CH11357.

\section{REFERENCE:}

[1] A. Gaidarzhy, M. Imboden, P. Mohanty, J. Rankin, and B. W. Sheldon, "High quality factor gigahertz frequencies in nanomechanical diamond resonators," Applied Physics Letters, vol. 91, pp. 203503-3, 2007.

[2] C. F. Wang, Y. S. Choi, J. C. Lee, E. L. Hu, J. Yang, and J. E. Butler, "Observation of whispering gallery modes in nanocrystalline diamond microdisks," Applied Physics Letters, vol. 90, p. 081110, 2007.

[3] H. M. Thomas, L. Xiao, H. H. Brian, W. B. Jeffrey, E. B. James, and F. Tatyana, "Low temperature internal friction in nanocrystalline diamond films," Applied Physics Letters, vol. 86, p. 081910, 2005.

[4] L. Sekaric, J. M. Parpia, H. G. Craighead, T. Feygelson, B. H. Houston, and J. E. Butler, "Nanomechanical resonant structures in nanocrystalline diamond," Applied Physics Letters, vol. 81, pp. 4455-4457, 2002.

[5] A. B. Hutchinson, P. A. Truitt, K. C. Schwab, L. Sekaric, J. M. Parpia, H. G. Craighead, and J. E. Butler, "Dissipation in nanocrystalline-diamond nanomechanical resonators," Applied Physics Letters, vol. 84, pp. 972-974, 2004.

[6] C. Liu, X. C. Xiao, J. Wang, B. Shi, V. P. Adiga, R. W. Carpick, J. A. Carlisle, and O. Auciello, "Dielectric properties of hydrogen-incorporated chemical vapor deposited diamond thin films," Journal of Applied Physics, vol. 102, p. 7, Oct 2007.

[7] O. Auciello, J. Birrell, J. A. Carlisle, J. E. Gerbi, X. C. Xiao, B. Peng, and H. D. Espinosa, "Materials science and fabrication processes for a new MEMS technoloey based on ultrananocrystalline diamond thin films," Journal of Physics-Condensed Matter, vol. 16, pp. R539-R552, Apr 2004.

[8] O. Auciello, S. Pacheco, A. V. Sumant, C. Gudeman, S. Sampath, A. Datta, R. W. Carpick, V. P. Adiga, P. Zurcher, M. Zhenqiang, Y. Hao-Chih, J. A. Carlisle, B. Kabius, J. Hiller, and S. Srinivasan, "Are Diamonds a MEMS' Best Friend?," Microwave Magazine, IEEE, vol. 8, pp. 61-75, 2007.

[9] N. N. Naguib, J. W. Elam, J. Birrell, J. Wang, D. S. Grierson, B. Kabius, J. M. Hiller, A. V. Sumant, R. W. Carpick, O. Auciello, and J. A. Carlisle, "Enhanced nucleation, smoothness and conformality of ultrananocrystalline diamond (UNCD) ultrathin films via tungsten interlayers," Chemical Physics Letters, vol. 430, pp. 345-350, 2006.

[10] U. Gysin, S. Rast, P. Ruff, E. Meyer, D. W. Lee, P. Vettiger, and C. Gerber, "Temperature dependence of the force sensitivity of silicon cantilevers," Physical Review B, vol. 69, p. 045403, 2004.

[11] V. P. Adiga, A. V. Sumant, S. Suresh, C. Gudeman, J. A. Carlisle, O. Auciello, and R. W. Carpick, "Mechanical stiffness and dissipation in ultrananocrystalline diamond resonators," Accepted for publication in Physical Review B, 2009.

[12] N. Sepulveda, L. Jing, D. M. Aslam, and J. P. Sullivan, "High-Performance Polycrystalline Diamond Micro- and Nanoresonators," Microelectromechanical Systems, Journal of, vol. 17, pp. 473-482, 2008.

[13] J. B. Wachtman, W. E. Tefft, D. G. Lam, and C. S. Apstein, "Exponential Temperature Dependence of Young's Modulus for Several Oxides," Physical Review, vol. 122, p. 1754, 1961.

[14] O. L. Anderson, "Derivation of Wachtman's Equation for the Temperature Dependence of Elastic Moduli of Oxide Compounds," Physical Review, vol. 144, p. 553, 1966.

[15] G. Mingxia, Z. Yichun, P. Likun, S. Zhuo, W. Shanzhong, and Q. S. Chang, "Temperature dependence of the elastic and vibronic behavior of $\mathrm{Si}, \mathrm{Ge}$, and diamond crystals," Journal of Applied Physics, vol. 102, p. 083524, 2007.

[16] H. J. McSkimin and P. Andreatch, Jr., "Elastic Moduli of Diamond as a Function of Pressure and Temperature," Journal of Applied Physics, vol. 43, pp. 2944-2948, 1972. 
[17] E. S. Zouboulis, M. Grimsditch, A. K. Ramdas, and S. Rodriguez, "Temperature dependence of the elastic moduli of diamond: A Brillouin-scattering study," Physical Review B, vol. 57, p. 2889, 1998.

[18] F. Szuecs, M. Werner, R. S. Sussmann, C. S. J. Pickles, and H. J. Fecht, "Temperature dependence of Young's modulus and degradation of chemical vapor deposited diamond," Journal of Applied Physics, vol. 86, pp. 6010-6017, 1999.

[19] C. Q. Sun, "Size dependence of nanostructures: Impact of bond order deficiency," Progress in Solid State Chemistry, vol. 35, pp. 1-159, 2007.

[20] M. X. Gu, Q. S. Chang, Z. Chen, T. C. A. Yeung, S. Li, C. M. Tan, and V. Nosik, "Size, temperature, and bond nature dependence of elasticity and its derivatives on extensibility, Debye temperature, and heat capacity of nanostructures," Physical Review B (Condensed Matter and Materials Physics), vol. 75, p. $125403,2007$.

[21] I. N. Remediakis, G. Kopidakis, and P. C. Kelires, "Softening of ultra-nanocrystalline diamond at low grain sizes," Acta Materialia, vol. 56, pp. 5340-5344, 2008.

[22] N. Sepulveda, D. M. Aslam, and J. P. Sullivan, "Polycrystalline Diamond RFMEMS Resonators with the Highest Quality Factors," in Micro Electro Mechanical Systems, 2006. MEMS 2006 Istanbul. 19th IEEE International Conference on, 2006, pp. 238-241.

[23] A. N. Cleland, Foundations of Nanomechanics. Heidelberg, New York: Springer Verlag, 2003.

[24] T. Tetsuya, K. Akihide, O. Fumiyasu, and T. Isao, "Debye temperature and stiffness of carbon and boron nitride polymorphs from first principles calculations," Physical Review B (Condensed Matter and Materials Physics), vol. 73, p. 064304, 2006.

[25] Y. H. Zhao and K. Lu, "Grain-size dependence of thermal properties of nanocrystalline elemental selenium studied by x-ray diffraction," Physical Review B, vol. 56, p. 14330, 1997.

[26] C. Seoanez, F. Guinea, and A. H. C. Neto, "Surface dissipation in nanoelectromechanical systems: Unified description with the standard tunneling model and effects of metallic electrodes," Physical Review B (Condensed Matter and Materials Physics), vol. 77, p. 125107, 2008.

[27] P. Mohanty, D. A. Harrington, K. L. Ekinci, Y. T. Yang, M. J. Murphy, and M. L. Roukes, "Intrinsic dissipation in high-frequency micromechanical resonators," Physical Review B, vol. 66, p. 085416, 2002.

[28] M. Imboden, P. Mohanty, A. Gaidarzhy, J. Rankin, and B. W. Sheldon, "Scaling of dissipation in megahertz-range micromechanical diamond oscillators," Applied Physics Letters, vol. 90, p. 3, Apr 2007. 